\title{
Methylation of TET2, CBL and CEBPA in Ph-negative myeloproliferative neoplasms
}

\author{
C S Chim, ${ }^{1}$ T S Wan, ${ }^{2}$ T K Fung, ${ }^{1}$ K F Wong ${ }^{3}$
}

${ }^{1}$ Department of Medicine, Queen Mary Hospital, University of Hong Kong, Hong Kong ${ }^{2}$ Department Pathology, Queen Mary Hospital, University of Hong Kong, Hong Kong ${ }^{3}$ Department of Pathology, Queen Elizabeth Hospital, Hong Kong

\section{Correspondence to}

Dr C S Chim, Department of Medicine, Queen Mary Hospital, The University of Hong Kong, Pokfulam Road, 852, Hong Kong; jcschim@hku.hk

Accepted 23 June 2010 Published Online First 29 July 2010

\section{ABSTRACT}

A loss-of-function mutation of TET2, CBL and CEBPA has been implicated in the pathogenesis or leukaemic transformation of myeloproliferative neoplasm. As tumour suppressor genes may potentially be inactivated by promoter hypermethylation, the authors studied the methylation status of these genes in three cell lines and diagnostic marrow samples from 45 patients with myeloproliferative neoplasm (MPN) (essential thrombocythaemia, $\mathrm{N}=34$; polycythaemia vera, $\mathrm{N}=7$ and primary myelofibrosis, $\mathrm{N}=4$ ) by methylation-specific PCR. TET2 was heterozygously methylated in MEG-01 and K562 but completely unmethylated in HEL. On the other hand, both CBL and CEBPA were completely unmethylated in all three cell lines. In the primary marrow samples, methylation of TET2 occurred in two (5.9\%) patients with essential thrombocythaemia $14.4 \%$ of all patients), both without JAK2 V617 mutation, but not in polycythaemia vera or primary myelofibrosis. There was no association between TET2 methylation with the type of MPN $(p=0.713)$. Hypermethylation of CBL or CEBPA was not detected in any patients. In summary, methylation of TET2, CBL and CEBPA is infrequent in MPN at diagnosis. The role of methylation of these genes at the time of leukaemic transformation warrants further study.

\section{INTRODUCTION}

Philadelphia-negative $(\mathrm{Ph}-\mathrm{ve})$ myeloproliferative neoplasm (MPN) is a stem cell disease with proliferation of myeloid compartment, leading to development of distinct clinical entities including polycythaemia vera (PV), essential thrombocythaemia (ET) and primary myelofibrosis (PMF). ${ }^{1-3}$ JAK2 V617F mutation, resulting in constitutive activation of JAK-STAT signalling, occurs in about half of the patients with ET and PMF but in more than $90 \%$ of patients with $\mathrm{PV}^{1}$ The recent discovery of JAK2 V617F in Ph-ve MPN is a major advance in the understanding of the pathogenesis of MPN, which is characterised by proliferation of the myeloid compartment resulting in distinct clinical entities.

Gene methylation is an alternative mechanism of gene inactivation, and various tumour suppressor genes regulating the cell cycle, apoptosis and cell signalling have been shown to be hypermethylated in haematological malignancies. ${ }^{4}$

Recently, inactivating mutations of several tumour suppressor genes have been detected in various myeloproliferative diseases or myeloid malignancies, including Casitas B lineage lymphoma (CBL), TET2 and CEBPA. CBL, which is localised to $11 \mathrm{q} 23$, possesses an E3 ubiquitinated ligase responsible for termination of activated tyrosine kinases. The tumour suppressor role of CBL has been demonstrated in mouse models, in which $\mathrm{c}-\mathrm{Cbl}^{-/-}$ mice displayed splenomegaly and an expanded haematopoietic progenitor pool, and accelerated blastic crisis when introduced into a BCR-ABL transgenic background. ${ }^{5}$ On the other hand, TET2 (TET oncogene family, member 2), localised to 4q24, is a putative tumour suppressor gene shown to be deleted in MDS/AML and was also shown to be mutated in $24(12 \%)$ of 198 patients with MPN (with or without the JAK2 V617F mutation). ${ }^{6}$ Finally, CCAAT/enhancer binding protein (C/EBP), $\alpha$, (CEBPA) which is localised to $19 \mathrm{q} 13$, is an important tumour suppressor gene regulating myeloid differentiation. Mutation, translocation and methylation of CEBPA have been demonstrated in AML. ${ }^{78}$ Moreover, knock-in mice with a targeted mutation in the CEBPA basic region, which specifically inhibits the CEBPA-E2F interaction, predisposed mice to a myeloproliferative disorder. ${ }^{9}$

As these three genes are putative tumour suppressors important in the pathogenesis of myeloid malignancies, we hypothesised that these genes might be inactivated by gene hypermethylation in patients with MPN.

\section{MATERIALS AND METHODS \\ Cell lines}

MEG-01 and K562 cells were kindly provided by Dr Yang Mo, Department of Paediatrics, Queen Mary

Table 1 Clinical demographics

\begin{tabular}{lc}
\hline & No of patients (\%) \\
\hline Myeloproliferative neoplasm type & $45(100 \%)$ \\
Polycythaemia vera & $7(15.6 \%)$ \\
Essential thrombocythaemia & $34(75.5 \%)$ \\
Primary myelofibrosis & $4(8.9 \%)$ \\
Gender & \\
Male & $24(53.3 \%)$ \\
Female & $21(46.7 \%)$ \\
Age (years) & \\
Median (range) & $67.5(28-89)$ \\
CBP & \\
Hb (g/dl) & $13.3(9-22)$ \\
Leucocyte count $\left(\times 10^{9} /\right)$ & $14.4(7-28)$ \\
Platelet count $\left(\times 10^{9} /\right)$ & $848(196-2275)$ \\
Symptoms at diagnosis* & \\
Asymptomatic & $25(62.5 \%)$ \\
Bleeding & $4(10 \%)$ \\
Erythromelalgia & $4(10 \%)$ \\
Stroke & $2(5 \%)$ \\
Abdominal pain & $3(7.5 \%)$ \\
Blurred vision & $1(2.5 \%)$ \\
Weight loss & $1(2.5 \%)$ \\
\hline
\end{tabular}

*Data available in 40 patients. CBP, complete blood picture. 
A

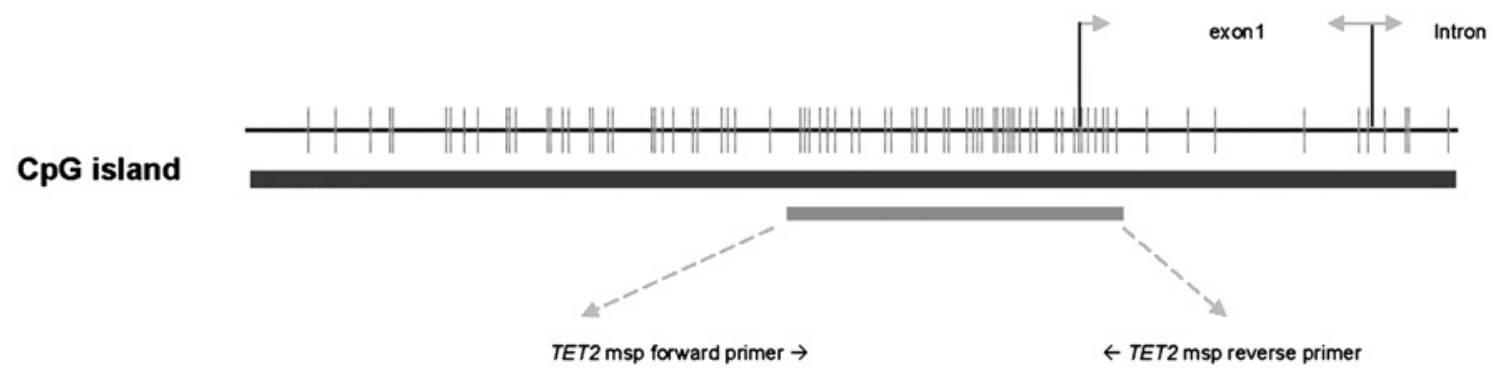

TET2 u-msp primer set: TET2 uf: 5'-TGGAGTGGGAGGAGGTTGGGGT-3'; ur: 5'-ATCTATTCTCATCACTCAACAAAACCA-3' ; product size: 211 bp

TET2 m-msp primer set: TET2 mf: 5'-CGGAGCGGGAGGAGGTCGGGGC-3'; mr: 5'-GTCTATTCTCATCACTCAACGAAACCG-3'; product size: 211bp

\section{TET2 m-msp sequencing}

Wild-type: TCGCGAG CGCCGCGCG C CC GG GT C C CGCT C GCAT GCAAGT CA CG T C C GC Methylated: $\quad$ T $\underline{c} G \underline{c} G A G \underline{c} G T \underline{C} G \underline{c} G \underline{c} G T T \underline{c} G G G T T$ T $\underline{c} G T T \underline{c} G T A T$ TTA A GT TA $\underline{c} G$ T T $\underline{c} G T$

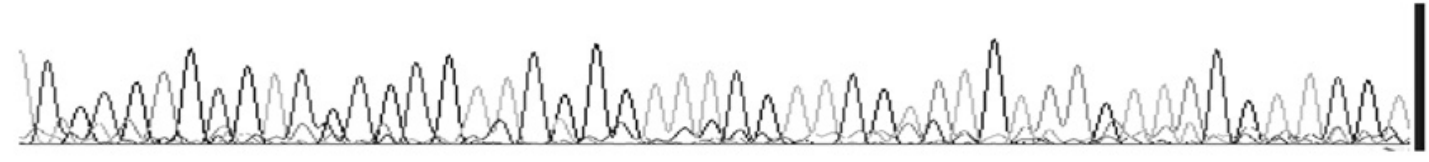

B

Methylation-specific PCR: TET2

M-MSP: donors

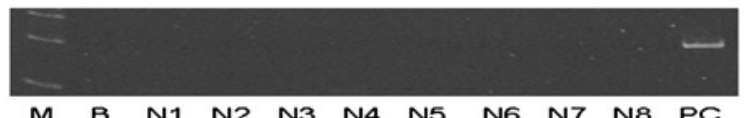

M-MSP: cell lines

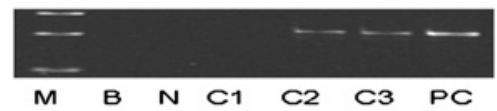

M-MSP: MPD samples

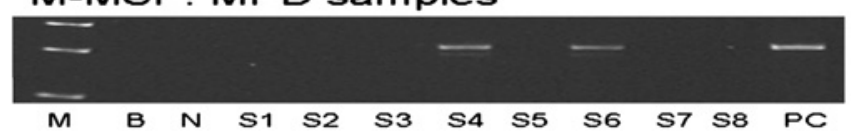

U-MSP: donors

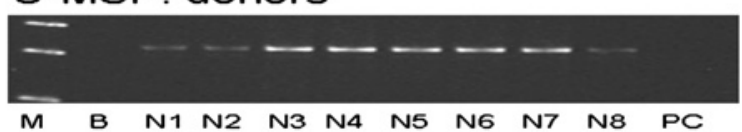

U-MSP: cell lines

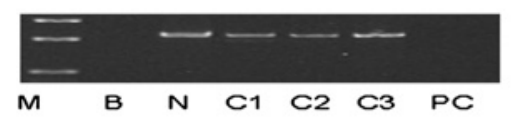

U-MSP MPD samples

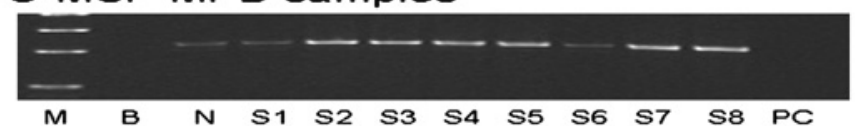

M: dna marker; B: reagent blank; N: normal control; PC: methylated control; S: MPD sample: C1: HEL; C2: MEGO1: C3: K562

C

TET2 m-msp MPD sample no. 42 sequencing
Methylated:

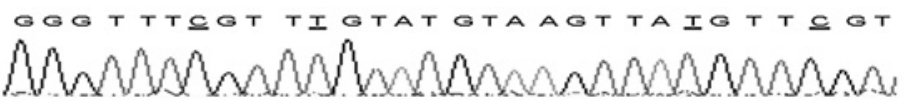

TET2 m-msp pc sequencing

WIIA-type: TCGCGAGCGCCGCGCGCCCGGGTCLCGCTCGCATGCAAGTCACGTCCOC

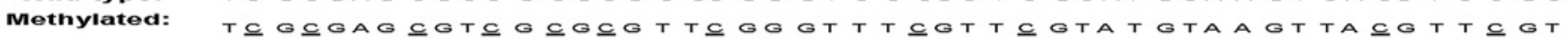

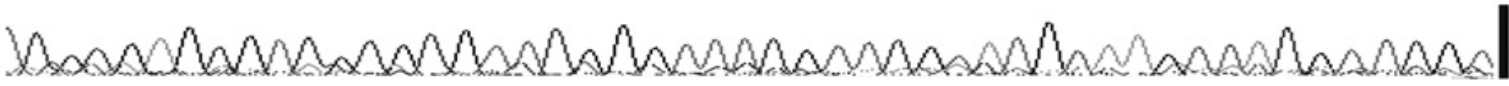

Figure 1 (A) CpG island of TET2 gene with location and sequence of methylation-specific polymerase chain reaction (MSP) primers (mf, forward MMSP primer; mr, reverse M-MSP primer; uf, forward U-MSP primer; ur, reverse U-MSP primer). (B) Direct sequencing of methylated positive control showing complete bisulfite conversion, and authenticity of MSP amplification. (C) M- and U-MSP of normal unmethylated controls (N1-N8), cell lines $(\mathrm{C} 1-\mathrm{C} 3)$ and primary marrow samples (S1-S8). M, molecular weight marker; NC, normal unmethylated control; PC, methylated positive control. 
Hospital, Hong Kong; and HEL from Dr Dong-Er Zhang, Department of Pathology and Molecular Biology, Moores Cancer Centre, University of California, San Diego, USA.

\section{Patient and diagnosis}

Diagnosis of MPN including ET, PV and PMF was based on WHO criteria. ${ }^{1}$ The clinical demographics of the patients are illustrated in table 1.

\section{Methylation-specific polymerase chain reaction (MSP)}

DNA was extracted from cell lines and primary bone marrow samples at diagnosis using a standard method. Using computer software, the presence of $\mathrm{CpG}$ islands at the corresponding gene promoters were determined, and unmethylated and methylated MSP primers were designed. Treatment of DNA with bisulfite for conversion of unmethylated cytosine to uracil (but unaffecting methylated cytosine) was performed with a commercially available kit (CpGenome DNA modification kit, Chemicon, New York). Methylation-specific PCR (MSP) was then validated in methylated (positive) and unmethylated (negative or normal) controls (DNA from normal cells) with methylated and unmethylated MSP primers.

\section{RESULTS}

Presence of a CpG island at the 5' untranslated regions (UTRs) for the all three genes was confirmed (figures 1-3).

A

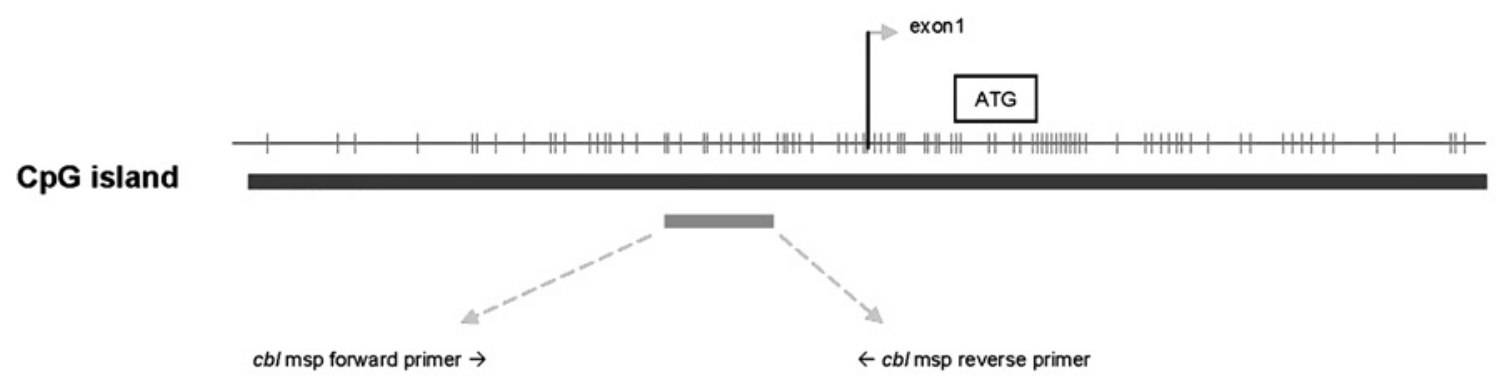

CBL u-msp primer set: CBL uf: 5'-TAGAGGGTGTAGGTTTAAAGGTTTT-3'; ur: 5'-AAACCAACTTACTAATAAACTCAAA-3' ; product size: 211bp

CBL m-msp primer set: CBL mf: 5'-AGGGCGTAGGTTTAAAGGTTTC-3'; mr: 5'-ACGAACCGACTTACTAATAAACTCG-3'; product size: 210bp

B

cblm-msp pc sequencing

Wild-type: CAC G C G AG CT G C TC G AA G CCC G T G GCC C G G GG ATC G T C GCT G C C CAG T C CG GCC G CG G C CG G CC G C

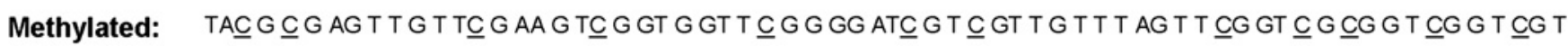

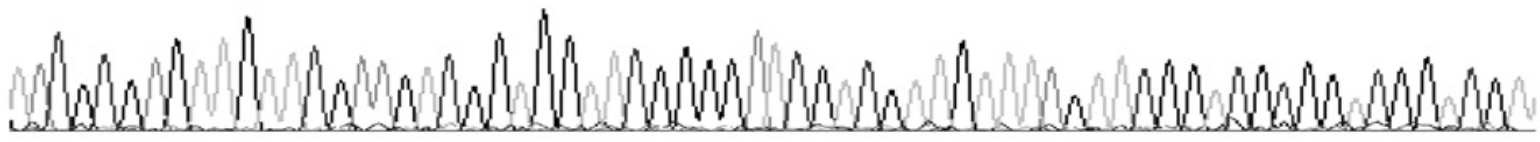

C

Methylation-specific PCR: CBL

\section{M-MSP: donors}

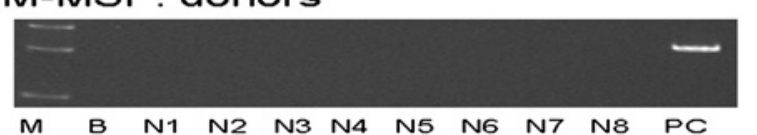

M-MSP: cell lines

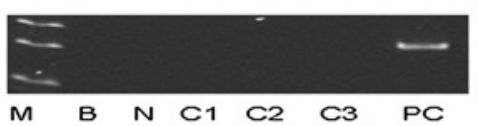

M-MSP: MPD samples

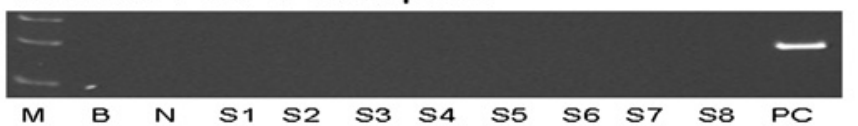

U-MSP: donors

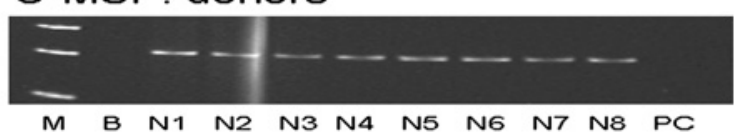

\section{U-MSP: cell lines}

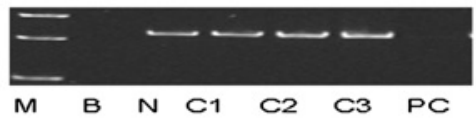

U-MSP: MPD samples

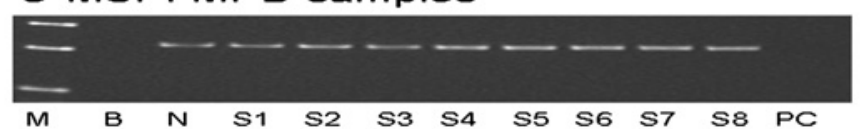

M: dna marker; B: reagent blank; N: normal control; PC: methylated control; S: MPD sample: C1: HEL; C2: MEGO1; C3: K562

Figure 2 (A) $\mathrm{CpG}$ island of CBL gene with location and sequence of methylation-specific polymerase chain reaction (MSP) primers (mf, forward MMSP primer; mr, reverse M-MSP primer; uf, forward U-MSP primer; ur, reverse U-MSP primer). (B) Direct sequencing of methylated positive control showing complete bisulfite conversion, and authenticity of MSP amplification. (C) M- and U-MSP of normal unmethylated controls (N1-N8), cell lines $(\mathrm{C} 1-\mathrm{C} 3)$ and primary marrow samples $(\mathrm{S} 1-\mathrm{S} 8)$. $\mathrm{M}$, molecular weight marker; NC, normal unmethylated control; PC, methylated positive control. 
Methylation-specific PCR (MSP) was then performed in methylated (positive) and unmethylated (negative or normal) controls (DNA from normal cells) with methylated and unmethylated MSP primers, which showed methylation of genes in the positive but not the normal DNA controls (figures 1-3). Direct sequencing of the methylated MSP of positive control confirmed specificity of the methylated amplification, in which methylated cytosine remained as cytosine upon sequencing (underlined) while unmethylated cytosine appeared as thymidine (figures 1-3). MSP was performed on cells from these three cell lines, HEL, MEG-01 and K562. HEL carried JAK2 V617F mutation, and MEG-01 and K562 were derived from blastic transformation of patients with chronic myeloid leukaemia. TET2 was heterozygously methylated in MEG-01 and K562

A

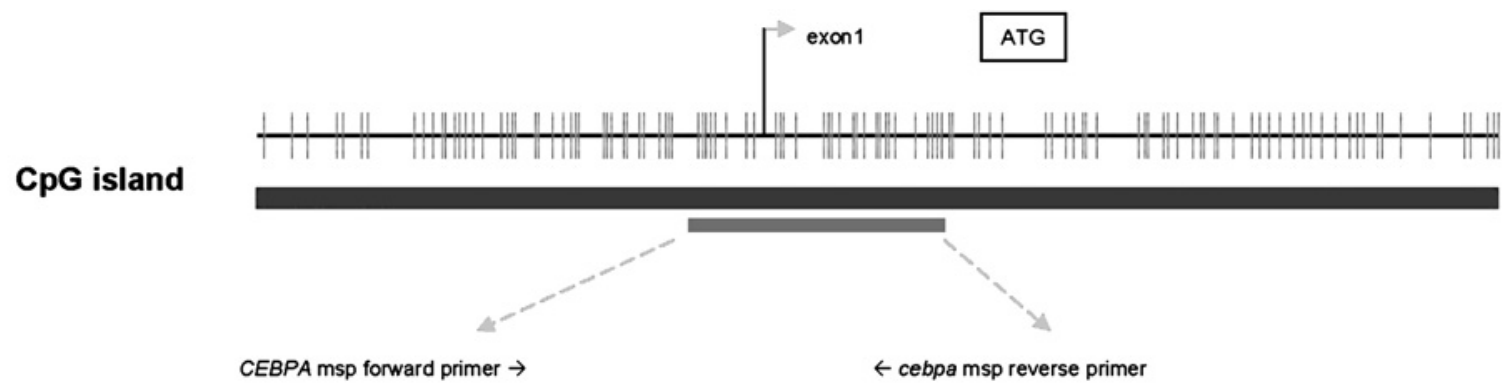

CEBPA u-msp primer set: CEBPA uf: 5'-AAGTTGGGTTGGTGTGGGTT-3'; ur: 5'-CCCAACATAACAAACCTCAACA-3' ; product size: 127bp

CEBPA m-msp primer set: CEBPA mf: 5'-AAGTTGGGTCGGCGCGGGTC-3'; mr: 5'-CCCGACATAACGAACCTCGACG-3'; product size: 127bp

B

\section{CEBPA m-msp pc sequencing}

Wild-type: C C GGGT G GGC G GC G GCGT C GCCC CGC G CA G GC TG G A GG CC GC C GA GGC T CG CCA T G C CG G Methylated:

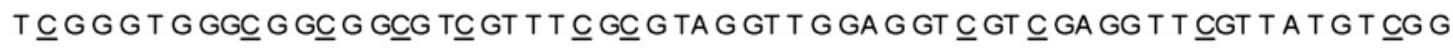

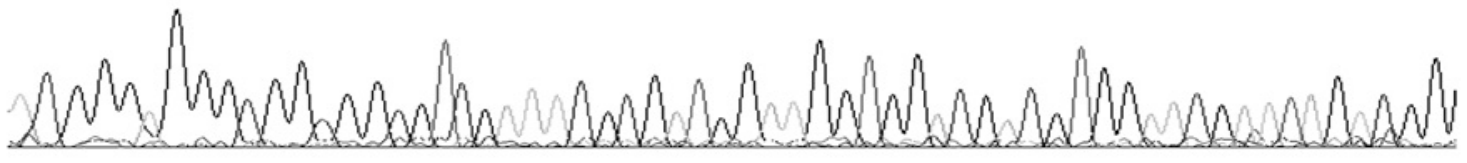

$\mathrm{C}$

\section{Methylation-specific PCR: CEBPA}

M-MSP: donors

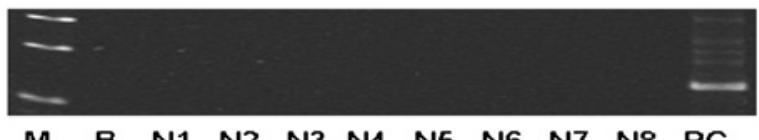

M-MSP: cell lines

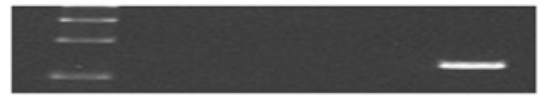

$\begin{array}{llllllll}M & B & \mathrm{~N} & \mathrm{C} 1 & \mathrm{C} 2 & \mathrm{C} 3 & \mathrm{PC}\end{array}$

\section{M-MSP: MPD samples}

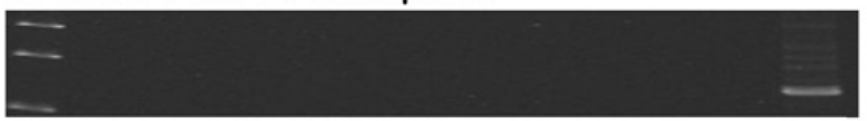

$\begin{array}{llllllllllll}M & B & N & S 1 & S 2 & S 3 & S 4 & \text { S5 } & \text { S6 } & \text { S7 } & \text { S8 } & \text { PC }\end{array}$

\section{U-MSP: donors}

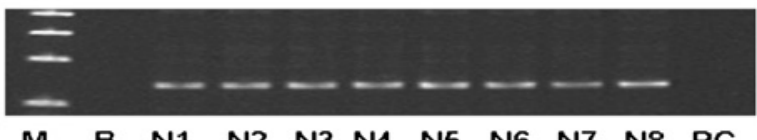

U-MSP: cell lines

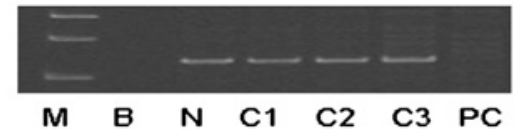

\section{U-MSP: MPD samples}

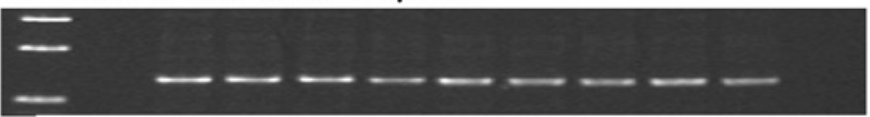

$\begin{array}{llllllllllll}M & B & N & S 1 & S 2 & S 3 & S 4 & \text { S5 } & \text { S6 } & \text { S7 } & \text { S8 } & \text { PC }\end{array}$

M: dna marker; B: reagent blank; N: normal control; PC: methylated control; S: MPD sample; C1: HEL; C2: MEG01; C3: K562

Figure 3 (A) CpG island of CEBPA gene with location and sequence of methylation-specific polymerase chain reaction (MSP) primers (mf, forward M-MSP primer; mr, reverse M-MSP primer; uf, forward U-MSP primer; ur, reverse U-MSP primer). (B) Direct sequencing of methylated positive control showing complete bisulfite conversion, and authenticity of MSP amplification. (C) M- and U-MSP of normal unmethylated controls (N1-N8), cell lines (C1-C3) and primary marrow samples (S1-S8). M, molecular weight marker; NC, normal unmethylated control; PC, methylated positive control. 


\section{Take-home messages}

- Loss-of-function mutation of TET2, CBL and CEBPA has been implicated in the pathogenesis or leukaemic transformation of MPN.

- Methylation of TET2, CBL and CEBPA is infrequent in MPN at diagnosis.

- The role of methylation of these genes at the time of leukaemic transformation warrants further study.

but completely unmethylated in HEL (figure 1). On the other hand, both CBL and CEBPA were completely unmethylated in all three cell lines (figures 2, 3).

In the primary diagnostic marrow samples, methylation of TET2 occurred in two $(5.9 \%)$ patients with ET (4.4\% of all patients) (figure 1), and none of the patients carried hypermethylation of CBL or CEBPA (figures 2, 3). These two patients did not carry a JAK2 V617F mutation. However, there was no association between TET2 methylation with the type of MPN $(p=0.713)$.

\section{DISCUSSION}

Recent studies strongly suggested that TET2 is a tumour suppressor gene as a TET2 mutation has been demonstrated to be acquired in $12-24 \%$ of myeloid neoplasms including AML, MDS, CMML and MPN. Moreover, while occasional patients possessed homozygous TET2 deletion, the majority showed a TET2 mutation together with gene deletion, thereby implicating a tumour suppressor role of TET2 in myeloid neoplasms. In particular, it is mutated in $12-13 \%$ of MPN including ET, PV and $\mathrm{PMF}^{6}{ }^{10}$ Therefore, we postulated that TET2 hypermethylation might occur. Indeed, it was demonstrated in two $(5.9 \%)$ patients in this cohort, and hence might be a possible mechanism to result in biallelic gene inactivation, and hence fulfil Knudson's hypothesis, which proposed that both alleles of a tumour suppressor gene have to be inactivated, usually by deletion and/or mutation, for complete abrogation of its function. ${ }^{11}$ The low frequency of TET2 gene hypermethylation was similar to the finding of absence of TET2 hypermethylation in another cohort of $354 \mathrm{MPN}$ patients. ${ }^{12}$ CBL is a tumour suppressor gene responsible for termination of receptorassociated tyrosine kinase signalling. The most common abnormality in myeloid neoplasms, in particular CMML, is the acquisition of uniparental disomy of a mutant allele of $\mathrm{CBL}$, leading to prolonged activation of tyrosine kinases after cytokine stimulation. ${ }^{5}$ In a study of CBL mutation in 577 patients with various haematological myeloproliferative disorders including 151 patients with Ph-ve MPN (74 PV, 53 PMF and $24 \mathrm{ET}$ ), the CBL mutation was detected in 26 patients. Three patients with PMF but none of those with PV or ET (ie, $2 \%$ of the $\mathrm{Ph}-\mathrm{ve}$ MPN patients) were found to carry $\mathrm{CBL}$ mutation. ${ }^{13}$ Our study showed that there was no methylation of CBL in MPN. Therefore, CBL mutation or methylation appeared infrequent in $\mathrm{Ph}-\mathrm{ve}$ MPNs.

Finally, CEBPA methylation has been shown in up to $51 \%$ of AML patients, ${ }^{14}$ and hence might be important in myeloid malignancy. It is one of the first reports of the absence of methylation of this gene in MPN patients. However, as inactivation of CEBPA might be responsible for leukaemic transformation, it will be important to study CEBPA hypermethylation in patients with leukaemia transforming from underlying MPN.

In summary, methylation of TET2, CBL and CEBPA is infrequent in MPN at diagnosis. The role of methylation of these genes at the time of leukaemic transformation warrants further study.

\section{Competing interests None.}

Patient consent Obtained.

Ethics approval Ethics approval was provided by the Institutional Review Board of Queen Mary Hospital.

Provenance and peer review Not commissioned; externally peer reviewed.

\section{REFERENCES}

1. Tefferi A, Thiele J, Orazi A, et al. Proposals and rationale for revision of the World Health Organization diagnostic criteria for polycythemia vera, essential thrombocythemia, and primary myelofibrosis: recommendations from an ad hoc international expert panel. Blood 2007;110:1092-7.

2. Chim CS, Kwong YL, Lie AK, et al. Long-term outcome of 231 patients with essential thrombocythemia: prognostic factors for thrombosis, bleeding, myelofibrosis, and leukemia. Arch Intern Med 2005;165:2651-8.

3. Chim CS, Kwong YL, Chan PT, et al. Polycythemia vera in Chinese patients: thirty-six years of experience. Am J Hematol 1997;56:59-62.

4. Chim CS, Liang R, Kwong YL. Hypermethylation of gene promoters in hematological neoplasia. Hematol Oncol 2002;20:167-76.

5. Sanada M, Suzuki T, Shih LY, et al. Gain-of-function of mutated C-CBL tumour suppressor in myeloid neoplasms. Nature 2009;460:904-8.

6. Delhommeau F, Dupont S, Della Valle V, et al. Mutation in TET2 in myeloid cancers N Engl J Med 2009;360:2289-301.

7. Chim CS, Wong AS, Kwong YL. Infrequent hypermethylation of CEBPA promotor in acute myeloid leukaemia. Br J Haematol 2002;119:988-90.

8. Koschmieder S, Halmos B, Levantini E, et al. Dysregulation of the C/EBPalpha differentiation pathway in human cancer. J Clin Oncol 2009;27:619-28.

9. Porse BT, Bryder D, Theilgaard-Mönch K, et al. Loss of C/EBP $\alpha$ cell cycle control increases myeloid progenitor proliferation and transforms the neutrophil granulocytic lineage. J Exp Med 2005;202:85-96.

10. Tefferi A, Pardanani A, Lim KH, et al. TET2 mutations and their clinical correlates in polycythemia vera, essential thrombocythemia and myelofibrosis. Leukemia 2009;23:905-11.

11. Sábado Alvarez C. Molecular biology of retinoblastoma. Clin Trans/ Oncol 2008;10:389-94.

12. Abdel-Wahab 0, Mullally A, Hedvat C, et al. Genetic characterization of TET1, TET2, and TET3 alterations in myeloid malignancies. Blood 2009;114:144-7.

13. Grand FH, Hidalgo-Curtis CE, Ernst T, et al. Frequent CBL mutations associated with $11 \mathrm{q}$ acquired uniparental disomy in myeloproliferative neoplasms. Blood 2009;113:6182-92.

14. Hackanson B, Bennett KL, Brena RM, et al. Epigenetic modification of CCAAT/ enhancer binding protein alpha expression in acute myeloid leukemia. Cancer Res 2008;68:3142-51. 


\title{
JCP
}

\section{Methylation of TET2, CBL and CEBPA in Ph-negative myeloproliferative neoplasms}

\author{
C S Chim, T S Wan, T K Fung, et al.
}

J Clin Pathol 2010 63: 942-946 originally published online July 29, 2010 doi: 10.1136/jcp.2010.080218

Updated information and services can be found at:

http://jcp.bmj.com/content/63/10/942.full.html

\begin{tabular}{|c|c|}
\hline & These include: \\
\hline References & $\begin{array}{l}\text { This article cites } 14 \text { articles, } 7 \text { of which can be accessed free at: } \\
\text { http://jcp.bmj.com/content/63/10/942.full.html\#ref-list-1 }\end{array}$ \\
\hline & $\begin{array}{l}\text { Article cited in: } \\
\text { http://jcp.bmj.com/content/63/10/942.full.html\#related-urls }\end{array}$ \\
\hline $\begin{array}{l}\text { Email alerting } \\
\text { service }\end{array}$ & $\begin{array}{l}\text { Receive free email alerts when new articles cite this article. Sign up in } \\
\text { the box at the top right corner of the online article. }\end{array}$ \\
\hline $\begin{array}{r}\text { Topic } \\
\text { Collections }\end{array}$ & $\begin{array}{l}\text { Articles on similar topics can be found in the following collections } \\
\text { Molecular genetics ( } 2142 \text { articles) }\end{array}$ \\
\hline
\end{tabular}

Notes

To request permissions go to:

http://group.bmj.com/group/rights-licensing/permissions

To order reprints go to:

http://journals.bmj.com/cgi/reprintform

To subscribe to BMJ go to:

http://group.bmj.com/subscribe/ 